\title{
Article \\ A New Family of Zeta Type Functions Involving the Hurwitz Zeta Function and the Alternating Hurwitz Zeta Function
}

\author{
Daeyeoul Kim ${ }^{1, *}$ and Yilmaz Simsek ${ }^{2}(\mathbb{D}$ \\ 1 Department of Mathematics and Institute of Pure and Applied Mathematics, Jeonbuk National University, \\ Jeonju 54896, Korea \\ 2 Department of Mathematics, Faculty of Science, University of Akdeniz, Antalya TR-07058, Turkey; \\ ysimsek@akdeniz.edu.tr \\ * Correspondence: kdaeyeoul@jbnu.ac.kr
}

check for updates

Citation: Kim, D.; Simsek, Y. A New Family of Zeta Type Function Involving the Hurwitz Zeta Function and the Alternating Hurwitz Zeta Function. Mathematics 2021, 9, 233. https://doi.org/10.3390/math9030233

Received: 14 December 2020 Accepted: 23 January 2021 Published: 25 January 2021

Publisher's Note: MDPI stays neutral with regard to jurisdictional clai$\mathrm{ms}$ in published maps and institutional affiliations.

Copyright: () 2021 by the authors. Licensee MDPI, Basel, Switzerland. This article is an open access article distributed under the terms and conditions of the Creative Commons Attribution (CC BY) license (https:// creativecommons.org/licenses/by/ $4.0 /)$.

\begin{abstract}
In this paper, we further study the generating function involving a variety of special numbers and ploynomials constructed by the second author. Applying the Mellin transformation to this generating function, we define a new class of zeta type functions, which is related to the interpolation functions of the Apostol-Bernoulli polynomials, the Bernoulli polynomials, and the Euler polynomials. This new class of zeta type functions is related to the Hurwitz zeta function, the alternating Hurwitz zeta function, and the Lerch zeta function. Furthermore, by using these functions, we derive some identities and combinatorial sums involving the Bernoulli numbers and polynomials and the Euler numbers and polynomials.
\end{abstract}

Keywords: Bernoulli numbers and polynomials; Euler numbers and polynomials; Apostol-Bernoulli and Apostol-Euler numbers and polynomials; Hurwitz-Lerch zeta function; Hurwitz zeta function; alternating Hurwitz zeta function; generating function; Mellin transformation

MSC: 05A15; 11B68; 26C0; 11M35

\section{Introduction}

The families of zeta functions and special numbers and polynomials have been studied widely in many areas. They have also been used to model real-world problems. In addition to these functions, generating functions for special numbers and polynomials have also been used commonly in variety of studies (cf. [1-34]).

The motivation of this paper is to apply the Mellin transformation to the following generating function:

$$
F_{\mathcal{Y}}(w, k, a)=\sum_{n=0}^{\infty} \mathcal{Y}_{n}(k, a) \frac{w^{n}}{n !}=\frac{a w}{e^{(k+1) w}-e^{-(k+1) w}+e^{w}-e^{-w}},
$$

where $k \in \mathbb{Z}$ and $a$, and $w \in \mathbb{R}$ (or $\mathbb{C}$ ) (cf. [25]). This yields a new family of zeta type functions involving the Hurwitz zeta function, the alternating Hurwitz zeta function, and the Lerch zeta function. After investigating some properties of these functions, we found that these functions can interpolate the Apostol-Bernoulli polynomials, the Bernoulli numbers and polynomials, and the Euler numbers and polynomials at negative integers.

The following notations, definitions and relations are used in this paper: Let $\mathbb{N}, \mathbb{Z}, \mathbb{Q}$, $\mathbb{R}$ and $\mathbb{C}$ denote the set of natural numbers, the set of integers, the set of rational numbers, the set of real numbers and the set of complex numbers, respectively.

$\mathbb{N}_{0}=\mathbb{N} \cup\{0\}$ and $\mathbb{Z}_{0}^{-}:=\mathbb{Z}^{-} \cup\{0\}, \mathbb{Z}^{-}:=\{-1,-2,-3, \cdots\}$.

Moreover, the falling factorial is given by:

$$
(x)_{n}=\left\{\begin{array}{ccc}
x(x-1)(x-2) \ldots(x-n+1) & \text { if } & n \in \mathbb{N} \\
1 & \text { if } & n=0
\end{array}\right.
$$


where $x \in \mathbb{R}$.

The Apostol-Bernoulli polynomials $\mathcal{B}_{n}(y ; \lambda)$ are defined by means of the following generating function:

$$
F_{A}(w, y ; \lambda)=\frac{w e^{w y}}{\lambda e^{w}-1}=\sum_{n=0}^{\infty} \mathcal{B}_{n}(y ; \lambda) \frac{w^{n}}{n !}
$$

(cf. [1]).

Substituting $y=0$ into (2), we have:

$$
\mathcal{B}_{0}(0 ; \lambda)=0
$$

and

$$
\lambda \mathcal{B}_{1}(1 ; \lambda)=1+\mathcal{B}_{1}(\lambda)
$$

and for $n \geq 2$,

$$
\lambda \mathcal{B}_{n}(1 ; \lambda)=\mathcal{B}_{n}(\lambda)
$$

(cf. [1]).

By using (2), we have:

$$
\mathcal{B}_{n}(y ; \lambda)=\sum_{j=0}^{n}\left(\begin{array}{l}
n \\
j
\end{array}\right) y^{n-j} \mathcal{B}_{j}(\lambda)
$$

(cf. [1]). By using (3), the first few values of the Apostol-Bernoulli polynomials are given as follows:

$$
\begin{aligned}
& \mathcal{B}_{1}(y ; \lambda)=\frac{1}{\lambda-1}, \mathcal{B}_{2}(y ; \lambda)=\frac{(\lambda-1) y-2 \lambda}{(\lambda-1)^{2}}, \\
& \mathcal{B}_{3}(y ; \lambda)=\frac{3(\lambda-1)^{2} y^{2}-6 \lambda(\lambda-1) y+3 \lambda(\lambda+1)}{(\lambda-1)^{3}},
\end{aligned}
$$

and so on.

Substituting $y=1$ into (2), we have the following Apostol-Bernoulli numbers:

$$
\mathcal{B}_{n}(1, \lambda)-\mathcal{B}_{n}(\lambda)=\sum_{j=0}^{n-1}\left(\begin{array}{l}
n \\
j
\end{array}\right) \mathcal{B}_{j}(\lambda),
$$

where

$$
\mathcal{B}_{n}(\lambda)=\mathcal{B}_{n}(0 ; \lambda) .
$$

Using (2), one has the following well-known identity:

$$
(1-\lambda) \mathcal{B}_{m}(\lambda)-\lambda \sum_{j=0}^{m-1}\left(\begin{array}{c}
m \\
j
\end{array}\right) \mathcal{B}_{j}(\lambda)=0
$$

(cf. $[24,29,31])$.

By using the above identity, we have the following few values of the Apostol-Bernoulli numbers:

$$
\mathcal{B}_{1}(\lambda)=\frac{1}{\lambda-1}, \mathcal{B}_{2}(\lambda)=\frac{-2 \lambda}{(\lambda-1)^{2}}, \mathcal{B}_{3}(\lambda)=\frac{3 \lambda(\lambda+1)}{(\lambda-1)^{3}}, \mathcal{B}_{4}(\lambda)=\frac{-4 \lambda\left(\lambda^{2}+4 \lambda+1\right)}{(\lambda-1)^{4}},
$$

and so on (cf. $[1,13,15,17-34])$. 
The Bernoulli polynomials $B_{n}(y)$ are defined by means of the following generating function:

$$
F_{B}(w, y)=\frac{w e^{w y}}{e^{w}-1}=\sum_{n=0}^{\infty} B_{n}(y) \frac{w^{n}}{n !}
$$

(cf. $[1,4-6,8,9,13-34])$.

Hence, a few values of the Bernoulli polynomials are given as follows:

$$
\begin{aligned}
& B_{0}(x)=1, B_{1}(x)=\frac{x-1}{2}, B_{2}(x)=\frac{x^{2}-x+1}{6}, B_{3}(x)=\frac{2 x^{3}-3 x^{2}+1 x}{2}, \\
& B_{4}(x)=\frac{30 x^{4}-60 x^{3}+30 x^{2}-1}{30}, B_{5}(x)=\frac{6 x^{5}-15 x^{4}+10 x^{3}-x}{6},
\end{aligned}
$$

and so on.

Since $B_{n}=B_{n}(0)$ denotes the Bernoulli numbers, a few values of these numbers are given as follows:

$$
B_{0}=1, B_{1}=-\frac{1}{2}, B_{2}=\frac{1}{6}, B_{3}=0, B_{4}=-\frac{1}{30}, B_{5}=0, B_{6}=\frac{1}{42}, B_{7}=0,
$$

and so on, here note that $B_{2 n+1}=0$ for $n \geq 2$ (cf. [1,4-6,8-10,13-32]).

The Apostol-Euler polynomials of the first kind $\mathcal{E}_{n}(x, \lambda)$ are defined by means of the following generating function:

$$
F_{P 1}(t, x ; k, \lambda)=\frac{2}{\lambda e^{t}+1} e^{t x}=\sum_{n=0}^{\infty} \mathcal{E}_{n}(x, \lambda) \frac{t^{n}}{n !},
$$

and by using (6), we have:

$$
\mathcal{E}_{n}(x ; \lambda)=\sum_{j=0}^{n}\left(\begin{array}{c}
n \\
j
\end{array}\right) x^{n-j} \mathcal{E}_{j}(\lambda)
$$

(cf. $[13,15,17-34])$.

By combining (6) with (2), we have the following well-known relation:

$$
(n+1) \mathcal{E}_{n}(x ; \lambda)+2 \mathcal{B}_{n+1}(x ;-\lambda)=0
$$

(cf. $[29,30])$.

Substituting $\lambda=1$ into (6), we have the Euler polynomials of the first kind; that is:

$$
E_{n}(x)=\mathcal{E}_{n}(x ; 1) .
$$

With the help of the Equation (7), a few values of the Euler polynomials of the first kind are given as follows:

$$
\begin{aligned}
& E_{0}(x)=1, E_{1}(x)=\frac{2 x-1}{2}, E_{2}(x)=x^{2}-x, E_{3}(x)=\frac{4 x^{3}-6 x^{2}+1}{4} \\
& E_{4}(x)=x^{4}-2 x^{3}+x, E_{5}(x)=\frac{2 x^{5}-5 x^{4}+5 x^{2}-1}{2}
\end{aligned}
$$

and so on (cf. [1,4-6,8,9,13-34]).

Putting $x=0$ in (6), we have the Apostol-Euler numbers:

$$
\mathcal{E}_{n}(\lambda)=\mathcal{E}_{n}(0, \lambda)
$$

where

$$
\mathcal{E}_{0}(\lambda)=\frac{2}{\lambda+1}
$$


By using (6), one has the following well-known identity:

$$
\mathcal{E}_{m}(\lambda)=-\frac{\lambda}{1+\lambda} \sum_{j=0}^{m-1}\left(\begin{array}{c}
m \\
j
\end{array}\right) \mathcal{E}_{j}(\lambda)
$$

(cf. $[24,29,31])$.

By using the above identity, we have the following few values of the Apostol-Euler numbers:

$$
\mathcal{E}_{1}(\lambda)=-\frac{2 \lambda}{(\lambda+1)^{2}}, \mathcal{E}_{2}(\lambda)=\frac{2 \lambda(\lambda-1)}{(\lambda+1)^{3}}, \mathcal{E}_{3}(\lambda)=-\frac{2 \lambda\left(\lambda^{2}-4 \lambda+1\right)}{(\lambda+1)^{4}},
$$

and so on. Setting $\lambda=1$ into (6), we have the Euler numbers of the first kind:

$$
E_{n}=\mathcal{E}_{n}^{(1)}(1)=E_{n}(0)
$$

which are given by:

$$
\frac{2}{e^{t}+1}=\sum_{n=0}^{\infty} E_{n} \frac{t^{n}}{n !}
$$

(cf. $[1,4-6,8,9,13-34]$ ). Hence a few of the values of the Euler numbers of the first kind are given as follows:

$$
E_{0}=1, E_{1}=-\frac{1}{2}, E_{2}=0, E_{3}=\frac{1}{4}, E_{5}=-\frac{1}{2}, E_{7}=\frac{17}{8}, E_{9}=-\frac{31}{2},
$$

and so on, here noting that $E_{2 n}=0$ for $n \geq 1$ (cf. [1,4-6,8-11,13-34]).

The Euler numbers of the second kind $E_{j}^{*}$ are given by means of the following generating function:

$$
\frac{2}{e^{t}+e^{-t}}=\sum_{j=0}^{\infty} E_{j}^{*} \frac{t^{j}}{j !}
$$

We now give the following well-known relation between the numbers $E_{n}^{*}$ and $E_{n}$ :

$$
\begin{aligned}
E_{n}^{*} & =2^{n} E_{n}\left(\frac{1}{2}\right) \\
& =\sum_{j=0}^{n}\left(\begin{array}{l}
n \\
j
\end{array}\right) 2^{j} E_{j} .
\end{aligned}
$$

Using the above equation, we have:

$$
E_{0}^{*}=1, E_{2}^{*}=-1, E_{3}^{*}=0, E_{4}^{*}=5, E_{5}^{*}=0, E_{6}^{*}=-61, E_{7}^{*}=0, E_{8}^{*}=1385,
$$

and so on, and here noting that $E_{2 n+1}^{*}=0$ for $n \geq 0$ (cf. [1,4-6,8-10,13-34]).

In [21], Simsek gave many remarks and observations on the numbers $E_{n}^{*}$ and $E_{n}$. Simsek [21] divided the Euler numbers into two groups, naming them as the Euler numbers of the first kind $E_{n}$ and the Euler numbers of the second kind $E_{n}^{*}$. The alternating Hurwitz zeta function can be used to interpolate these numbers at negative integers.

By using Equation (1), we have:

$$
F_{\mathcal{Y}}(w, k, a)=\frac{a w e^{(k+1) w}}{\left(e^{(k+2) w}-1\right)\left(e^{k w}+1\right)}
$$

(cf. [25]). 
Equation (10) yields the following relations among the numbers $\mathcal{Y}_{n}(k, a)$, the Bernoulli polynomials and numbers, and the Euler polynomials and numbers:

$$
\begin{gathered}
\mathcal{Y}_{n}(0,2)=2^{n-1} B_{n}\left(\frac{1}{2}\right)=\frac{1}{2} \sum_{j=0}^{n}\left(\begin{array}{l}
n \\
j
\end{array}\right) B_{j}(1) E_{n-j}, \\
\mathcal{Y}_{n}(1,2)=\sum_{j=0}^{n}\left(\begin{array}{l}
n \\
j
\end{array}\right) 3^{n-j-1} B_{n-j}\left(\frac{2}{3}\right) E_{j},
\end{gathered}
$$

and

$$
\mathcal{Y}_{n}(1,2)=\sum_{j=0}^{n}\left(\begin{array}{l}
n \\
j
\end{array}\right) 3^{n-j-1} B_{n-j} E_{j}(2),
$$

(cf. [25]). By using (1), we arrive at the following theorem:

Theorem 1. (cf. [25]) Let $n \in \mathbb{N}_{0}$. Then we have:

$$
\begin{aligned}
& (\mathcal{Y}(k, a)+k+1)^{n}-(\mathcal{Y}(k, a)-k-1)^{n}+(\mathcal{Y}(k, a)+1)^{n}-(\mathcal{Y}(k, a)-1)^{n} \\
= & \begin{cases}0 & \text { if } n \neq 1 \\
a & \text { if } n=1\end{cases}
\end{aligned}
$$

where, after expansion, each index of $\mathcal{Y}^{n}(k, a)$ is to be replaced by the corresponding $a \in \mathbb{R}$ and $k \in \mathbb{Z}$ as in the usual umbral convention method and the finite difference methods.

Using (11), we have the following few values of the numbers $\mathcal{Y}_{n}(k, a)$ :

$$
\mathcal{Y}_{0}(k, a)=\frac{a}{2(k+1)}, \mathcal{Y}_{1}(k, a)=0, \mathcal{Y}_{2}(k, a)=-\frac{a(k+1)^{3}+a}{6(k+1)^{2}}
$$

and so on.

Theorem 2. (cf. [25]) Let $n \in \mathbb{N}_{0}$. Then we have:

$$
\mathcal{Y}_{n}(k, a)=\frac{a}{2} \sum_{v=0}^{n}\left(\begin{array}{l}
n \\
v
\end{array}\right) k^{n-v}(k+2)^{v-1} B_{v} E_{n-v}\left(\frac{k+1}{k}\right) .
$$

Theorem 3. (cf. [25]) Let $n \in \mathbb{N}_{0}$. Then we have:

$$
\mathcal{Y}_{n}(k, a)=\frac{a}{2} \sum_{v=0}^{n}\left(\begin{array}{l}
n \\
v
\end{array}\right) k^{n-v}(k+2)^{v-1} E_{n-v} B_{v}\left(\frac{k+1}{k+2}\right) .
$$

Identities given by Equations (12) and (13) will be used in the next section.

\section{Family of Zeta Functions}

In order to present the results of this paper, we also need the following Lerch zeta function (the Hurwitz-Lerch zeta function) $\Phi(\lambda, z, b)$, which is a generalization of the Hurwitz zeta function, the polylogarithm, and the Riemann zeta function. This function was discovered by the Czech mathematician Mathias Lerch (20 February 1860-3 August 1922) (cf. $[1,4,6,27,28,30,31])$.

Let $z \in \mathbb{C} . z=x+i y$ with $x, y \in \mathbb{R}$.

The Hurwitz-Lerch zeta function $\Phi(\lambda, z, b)$ is defined by:

$$
\Phi(\lambda, z, b)=\sum_{j=0}^{\infty} \frac{\lambda^{j}}{(j+b)^{z}}
$$

where $b \in \mathbb{C} \backslash \mathbb{Z}_{0}^{-} ; \lambda, z \in \mathbb{C}$ when $|\lambda|<1$; and $x>1$ when $|\lambda|=1$ (cf. $[1,4,6,27,28,30,31]$ ). 
The function $\Phi(\lambda, z, b)$ interpolates the Apostol-Bernoulli polynomials at negative integers:

$$
\Phi(\lambda, 1-n, b)=-\frac{1}{n} \mathcal{B}_{n}(b ; \lambda),
$$

where $n \in \mathbb{N},|\lambda|<1$ (cf. [1,11,27,28,30,31,33]).

By combining (14) with (8), we have the following known result:

$$
\Phi(-\lambda, 1-n, b)=\frac{1}{2} \mathcal{E}_{n-1}(b ; \lambda)
$$

where $n \in \mathbb{N}$.

Putting $|\lambda|=1$ in (15), the function $\Phi(\lambda, z, b)$ reduces to the following Hurwitz zeta function $\zeta(z, b)$ :

$$
\zeta(z, b)=\sum_{j=0}^{\infty} \frac{1}{(j+b)^{z}}
$$

where $(z \in \mathbb{C}$ and $z=x+i y$ with $x, y \in \mathbb{R}) x>1$.

The function $\zeta(z, b)$ interpolates the Bernoulli polynomials at negative integers:

$$
\zeta(1-n, b)=-\frac{1}{n} B_{n}(b),
$$

where $n \in \mathbb{N}$ (cf. $[4,7,8,15,18,26-32,34])$.

Substituting $\lambda=-1$ into the function $\Phi(\lambda, z, b)$, we have the following well-known alternating Hurwitz zeta function $\zeta_{E}(z, b)$ :

$$
\zeta_{E}(z, b)=\sum_{j=0}^{\infty} \frac{(-1)^{j}}{(j+b)^{z}}
$$

where $(z \in \mathbb{C}$ and $z=x+i y$ with $x, y \in \mathbb{R}) x>1$ and $b>0$.

The function $\zeta_{E}(z, b)$ interpolates the Euler polynomials at negative integers:

$$
\zeta_{E}(-n, b)=\frac{(-1)^{n}}{2} E_{n}(1-b),
$$

where $b \in \mathbb{N}_{0}$ (cf. [4]) [Equation (3.20)].

In this study, we find it to be convenient to make use of the notation $\zeta_{E}(z, b)$, instead of their traditionally used notations $\eta(z, b)$ given by various studies (see, for example, $[4,6,8,27,28,30,31])$ :

$$
\zeta_{E}(z, b):=\eta(z, b):=\Phi(-1, z, b) .
$$

Here we note that the alternating Hurwitz zeta function is also the so-called the HurwitzEuler eta function (cf. $[4,8,31,34])$.

\section{A New Family of Zeta Type Functions}

There are many well-known methods to construct zeta type functions (cf. [1-34]). Here, our method relies on an application the Mellin transform to the generating function for the numbers $\mathcal{Y}_{n}(k, a)$.

In order to give this new family of zeta type functions, we assume that $s \in \mathbb{C}$ with $s=c+i d, i^{2}=-1$, and $c>1$.

By applying the Mellin transformation to the generating function (10), we arrive at the following integral representation of a new family of zeta type functions $\mathcal{Z}(s ; k, a)$ :

$$
\mathcal{Z}(s ; k, a)=\frac{1}{\Gamma(s)} \int_{0}^{\infty} w^{s-1} F_{\mathcal{Y}}(-w, k, a) d w .
$$


Hence,

$$
\mathcal{Z}(s ; k, a)=\frac{a}{\Gamma(s)} \int_{0}^{\infty} \frac{w^{s} e^{-w(k+1)}}{\left(e^{-w(k+2)}-1\right)\left(e^{-k w}+1\right)} d w .
$$

where $(s \in \mathbb{C}$ with $s=c+i d, c, d \in \mathbb{R}) c>1$. Assuming that $\left|e^{-w(k+2)}\right|<1$ and $\left|e^{-w k}\right|<1$, the above equation reduces to the following relation:

$$
\mathcal{Z}(s ; k, a)=\frac{a}{\Gamma(s)} \sum_{m=0}^{\infty} \sum_{n=0}^{\infty}(-1)^{n} \int_{0}^{\infty} w^{s} e^{-w(k n+(k+2) m+k+1)} d w
$$

with:

$$
\min \{c, k n+(k+2) m+k+1\}>0,
$$

where the supplementary restriction $k n+(k+2) m+k+1>0$ is required for the convergence of the infinite integral occurring on the right-hand side at its upper terminal of Equation (19). After some elementary calculation, we get:

$$
\mathcal{Z}(s ; k, a)=a \sum_{n=0}^{\infty} \sum_{m=0}^{\infty} \frac{(-1)^{n}}{(k+1+k n+(k+2) m)^{s+1}} \frac{1}{\Gamma(s)} \int_{0}^{\infty} z^{s} e^{-z} d z,
$$

where $(s \in \mathbb{C}$ with $s=c+i d, c, d \in \mathbb{R}) c>1$, and $k+1+k n+(k+2) m>0$.

Therefore, with the aid of the Euler gamma function, the following equation is easily reached applying the partial integral to the integral on the right-hand side of the above equation:

$$
\mathcal{Z}(s ; k, a)=a \frac{\Gamma(s+1)}{\Gamma(s)} \sum_{n=0}^{\infty} \sum_{m=0}^{\infty} \frac{(-1)^{n}}{(k+1+k n+(k+2) m)^{s+1}} .
$$

Since

$$
\Gamma(s+1)=s \Gamma(s),
$$

we arrive at the following definition:

Definition 1. Let $s \in \mathbb{C}$ with $s=c+i d$ and let $k \in \mathbb{N}, a, c, d \in \mathbb{R}$ with $c>1$. A new family of zeta functions involving the alternating Hurwitz zeta function and the Hurwitz zeta function is defined by:

$$
\mathcal{Z}(s ; k, a)=a s \sum_{n=0}^{\infty} \sum_{m=0}^{\infty} \frac{(-1)^{n}}{(k+1+k n+(k+2) m)^{s+1}} .
$$

Here, we show that the function $\mathcal{Z}(s ; k, a)$ is related to not only to the Hurwitz zeta function and the alternating Hurwitz zeta function, but also to the Lerch zeta function.

We now give the following two alternative forms of the function $\mathcal{Z}(s ; k, a)$ :

I. Combining (20) with (16), we obtain:

$$
\mathcal{Z}(s ; k, a)=\frac{a s}{(k+2)^{s+1}} \sum_{n=0}^{\infty}(-1)^{n} \zeta\left(s+1, \frac{k(n+1)+1}{k+2}\right) .
$$

II. Combining (20) with (18), we get:

$$
\mathcal{Z}(s ; k, a)=\frac{a s}{k^{s+1}} \sum_{m=0}^{\infty} \zeta_{E}\left(s+1, \frac{k+1+(k+2) m}{k}\right) .
$$

By applying the principle of analytic continuation together with the Cauchy Residue Theorem to (19) and making use of the same method as that used earlier by Srivastava et al. (see, for details, [32]), we arrive at the following theorems: 
Theorem 4. Let $v \in \mathbb{N}$. Then we have:

$$
\mathcal{Z}(-v ; k, a)=-a \sum_{j=0}^{v}\left(\begin{array}{l}
v \\
j
\end{array}\right) \frac{k^{j}(k+2)^{v-j-1}}{j+1} B_{v-j} \mathcal{B}_{j+1}\left(\frac{k+1}{k} ;-1\right) .
$$

Proof. Substituting $s=-v,(v \in \mathbb{N})$ into (21), we get:

$$
\mathcal{Z}(-v ; k, a)=-v a(k+2)^{v-1} \sum_{n=0}^{\infty}(-1)^{n} \zeta\left(1-v, \frac{k(n+1)+1}{k+2}\right) .
$$

Combining the above equation with (17), we obtain:

$$
\mathcal{Z}(-v ; k, a)=a(k+2)^{v-1} \sum_{n=0}^{\infty}(-1)^{n} B_{v}\left(\frac{k(n+1)+1}{k+2}\right) .
$$

After some elementary calculations in the above equation, we have:

$$
\mathcal{Z}(-v ; k, a)=a(k+2)^{v-1} \sum_{j=0}^{v}\left(\begin{array}{l}
v \\
j
\end{array}\right)\left(\frac{k}{k+2}\right)^{j} B_{v-j} \sum_{n=0}^{\infty}(-1)^{n}\left(n+\frac{k+1}{k}\right)^{j} .
$$

Combining the above equation with Equation (14), which modified as follows:

$$
\Phi\left(-1,-j, \frac{k+1}{k}\right)=\sum_{n=0}^{\infty}(-1)^{n}\left(n+\frac{k+1}{k}\right)^{j}=-\frac{1}{j+1} \mathcal{B}_{j+1}\left(\frac{k+1}{k} ;-1\right)
$$

we obtain:

$$
\mathcal{Z}(-v ; k, a)=-a \sum_{j=0}^{v}\left(\begin{array}{l}
v \\
j
\end{array}\right) \frac{k^{j}(k+2)^{v-j-1}}{j+1} B_{v-j} \mathcal{B}_{j+1}\left(\frac{k+1}{k} ;-1\right) .
$$

Thus the proof of theorem is completed.

Substituting $x=\frac{k+1}{k}$ into (8), we have:

$$
\mathcal{B}_{j+1}\left(\frac{k+1}{k} ;-1\right)=-\frac{j+1}{2} \mathcal{E}_{j}\left(\frac{k+1}{k} ; 1\right) .
$$

Since

$$
\mathcal{E}_{j}\left(\frac{k+1}{k} ; 1\right)=E_{j}\left(\frac{k+1}{k}\right)
$$

we get:

$$
\mathcal{B}_{j+1}\left(\frac{k+1}{k} ;-1\right)=-\frac{j+1}{2} E_{j}\left(\frac{k+1}{k}\right) .
$$

Combining the above equation with (23), we arrive at the following theorems:

Theorem 5. Let $v \in \mathbb{N}$. Then we have:

$$
\mathcal{Z}(-v ; k, a)=\frac{a}{2} \sum_{j=0}^{v}\left(\begin{array}{l}
v \\
j
\end{array}\right) k^{j}(k+2)^{v-j-1} B_{v-j} E_{j}\left(\frac{k+1}{k}\right) .
$$

Theorem 6. Let $v \in \mathbb{N}$. Then we have:

$$
\mathcal{Z}(-v ; k, a)=\frac{a v}{2} \sum_{j=0}^{v-1}\left(\begin{array}{c}
v-1 \\
j
\end{array}\right) \frac{k^{v-j-1}(k+2)^{j}}{j+1} E_{v-j-1} B_{j+1}\left(\frac{k+1}{k+2}\right) .
$$


Proof. Substituting $s=-v,(v \in \mathbb{N})$ into (22), we get:

$$
\mathcal{Z}(-v ; k, a)=-a v k^{v-1} \sum_{m=0}^{\infty} \zeta_{E}\left(1-v, \frac{k+1+(k+2) m}{k}\right) .
$$

Applying Equation (24) to the above equation, and using (25), we obtain:

$$
\mathcal{Z}(-v ; k, a)=-\frac{a v k^{v-1}}{2} \sum_{m=0}^{\infty} E_{v}\left(\frac{k+1+(k+2) m}{k}\right) .
$$

Therefore:

$$
\mathcal{Z}(-v ; k, a)=-\frac{a v}{2} \sum_{j=0}^{v-1}\left(\begin{array}{c}
v-1 \\
j
\end{array}\right) k^{v-j-1}(k+2)^{j} E_{v-j-1} \sum_{m=0}^{\infty}\left(m+\frac{k+1}{k+2}\right)^{j} .
$$

By using Equation (17), we have:

$$
\zeta\left(-j, \frac{k+1}{k+2}\right)=\sum_{m=0}^{\infty}\left(m+\frac{k+1}{k+2}\right)^{j}=-\frac{1}{j+1} B_{j+1}\left(\frac{k+1}{k+2}\right)
$$

Combining the above equation with (28), after some calculations, we arrive at the desired result.

By using (27), for $v \in \mathbb{N}$, we also obtain:

$$
\mathcal{Z}(-v ; k, a)=\frac{a}{2} \sum_{j=0}^{v-1}\left(\begin{array}{c}
v \\
j+1
\end{array}\right) k^{v-j-1}(k+2)^{j} E_{v-j-1} B_{j+1}\left(\frac{k+1}{k+2}\right) .
$$

Remark 1. With the help of the theorems given above, we have examined the behavior of the function $\mathcal{Z}(s ; k, a)$. In addition, it was seen that the numbers $B_{v-j} \mathcal{B}_{j+1}\left(\frac{k+1}{k} ;-1\right), B_{v-j} E_{j}\left(\frac{k+1}{k}\right)$ and $E_{v-j} B_{j}\left(\frac{k+1}{k+2}\right)\left(v, j \in \mathbb{N}_{0}\right.$ with $\left.v \geq j\right)$ are also interpreted by the function $\mathcal{Z}(s ; k, a)$, respectively, at negative integers.

Combining (29) with (12), we arrive at the following corollary:

Corollary 1. Let $v \in \mathbb{N}$. Then we have:

$$
\mathcal{Z}(-v ; k, a)=\mathcal{Y}_{v}(k, a)
$$

By combining (30) with (23) and (29), we get the following result:

Theorem 7. Let $v \in \mathbb{N}$. Then we have:

$$
\begin{aligned}
& \sum_{j=0}^{v-1}\left(\begin{array}{c}
v \\
j+1
\end{array}\right) k^{v-j-1}(k+2)^{j} E_{v-j-1} B_{j+1}\left(\frac{k+1}{k+2}\right) \\
= & \sum_{j=0}^{v}\left(\begin{array}{c}
v \\
j
\end{array}\right) k^{j}(k+2)^{v-j-1} B_{v-j} E_{j}\left(\frac{k+1}{k}\right) .
\end{aligned}
$$

\section{Conclusions}

In this paper, we defined a new class zeta type function. By applying the Mellin transformation to Equation (1), we presented an integral representation of this function. With the help of this integral representation, we also showed that this function interpolates the products of the Apostol-Bernoulli polynomials, the Bernoulli polynomials and numbers, and also Euler polynomials and numbers at negative integers. For the proof of the main 
theorems of this paper, we used the Lerch zeta function, the Hurwitz zeta function, and the alternating Hurwitz zeta function. We also gave relations among the Apostol-Bernoulli polynomials, Apostol-Euler polynomials, a combinatorial sum involving the Bernoulli numbers and polynomials, and the Euler numbers and polynomials.

Here, we also note that in [12], Kucukoglu and Simsek gave some formulas involving

$$
h(x ; n, m)=B_{n} \mathcal{B}_{m}(x)
$$

by using a zeta type function, the Dirichlet series, and Lambert-type series. The method of this paper is also very useful in order to obtain identities including the function $h(x ; n, m)$.

The results given in this paper can be potentially used in physics and engineering applications, and especially in mathematics.

Our future plan is to study new types of $L$-functions, Dirichlet type series and their applications with the help of the function $\mathcal{Z}(s ; k, a)$. We will also investigate relationships among this function, special polynomials, and splines containing Bernstein basis functions.

Author Contributions: All authors conceived of the study, participated in its design, and read and approved the final manuscript.

Funding: The present investigation was supported by the Scientific Research Project Administration of Akdeniz University (Project ID: FBA-2020-5299), and the first author was supported by the selection as a research-oriented professor of Jeonbuk National University in 2020 and the Basic Science Research Program through the National Research Foundation of Korea (NRF) funded by the Ministry of Education (NRF-2018R1D1A1B07041132).

Institutional Review Board Statement: Not applicable.

Informed Consent Statement: Not applicable.

Data Availability Statement: Not applicable.

Acknowledgments: The authors would like to thank the referees for their valuable comments on the present paper.

Conflicts of Interest: The authors declare no conflict of interest.

\section{References}

1. Apostol, T.M. On the Lerch Zeta function. Pac. J. Math. 1951, 1, 161-167. [CrossRef]

2. Bayad, A.; Simsek, Y. Values of twisted Barnes zeta functions at negative integers. Russ. J. Math. Phys. 2013, $20,129-137$. [CrossRef]

3. Bayad, A.; Simsek, Y. Note on the Hurwitz Zeta Function of Higher Order. AIP Conf. Proc. 2011, 1389, 389-391. [CrossRef]

4. Choi, J.; Srivastava, H.M. The multiple Hurwitz zeta function and the multiple Hurwitz-Euler eta function. Taiwan. J. Math. 2011, 15, 501-522. [CrossRef]

5. Comtet, L. Advanced Combinatorics; D. Reidel Publishing Company: Dordrecht, The Netherlands, 1974.

6. Erdelyi, A. Higher Transcendental Functions; Bateman, H., Ed.; McGraw-Hill Book Company: New York, NY, USA, 1953; Volumes I-III.

7. Hwang, K.-W.; Ryoo, C.S. Some Properties for Multiple Twisted $(p, q)-$ L-Function and Carlitz's Type Higher-Order Twisted $(p, q)$-Euler Polynomials. Mathematics 2019, 7, 1205. [CrossRef]

8. Hu, S.; Kim, D.; Kim, M.-S. Special values and integral representations for the Hurwitz-type Euler zeta functions. arxiv 2015, arXiv:1508.04084.

9. Kim, T.; Rim, S.H.; Simsek, Y.; Kim, D. On the analogs of Bernoulli and Euler numbers, related identities and zeta and $l$-functions. J. Korean Math. Soc. 2008, 45, 435-453. [CrossRef]

10. Kruchinin, D.; Kruchinin, V.; Simsek, Y. Generalized Tepper's identity and its application. Mathematics 2020, 8, 243. [CrossRef]

11. Kucukoglu, I.; Simsek, Y.; Srivastava, H.M. A new family of Lerch-type zeta functions interpolating a certain class of higher-order Apostol-type numbers and Apostol-type polynomials. Quaest. Math. 2019, 42, 465-478. [CrossRef]

12. Kucukoglu, I.; Simsek, Y. Identities for Dirichlet and Lambert-type series arising from the numbers of a certain special word. Appl. Anal. Discret. Math. 2019, 13, 787-804. [CrossRef]

13. Luo, Q.M.; Srivastava, H.M. Some generalizations of the Apostol-Genocchi polynomials and the Stirling numbers of the second kind. Appl. Math. Comput. 2011, 217, 5702-5728. [CrossRef]

14. Milne-Thomson, L.M. The Calculus of Finite Differences; Macmillan and Co.: London, UK, 1933. 
15. Ozden, H.; Simsek, Y. Modification and unification of the Apostol-type numbers and polynomials and their applications. Appl. Math. Comput. 2014, 235, 338-351. [CrossRef]

16. Roman, S. The Umbral Calculus; Dover Publ. Inc.: New York, NY, USA, 2005.

17. Simsek, Y. q-Analogue of the twisted $l$-series and $q$-twisted Euler numbers. J. Number Theory 2005, 110, 267-278. [CrossRef]

18. Simsek, Y. On twisted $q$-Hurwitz zeta function and q-two-variable L-function. Appl. Math. Comput. 2007, 187, 466-473. [CrossRef]

19. Simsek, Y. q-Hardy Berndt type sums associated with $q$-Genocchi type zeta and $q$-l-functions. Nonlinear Anal. 2009, 71, e377-e395. [CrossRef]

20. Simsek, Y. Twisted $p$-adic $(h, q)$-L-functions. Comput. Math. Appl. 2010, 59, 2097-2110. [CrossRef]

21. Simsek, Y. Special functions related to Dedekind-type DC-sums and their applications. Russ. J. Math. Phys. 2010, 17, 495-508. [CrossRef]

22. Simsek, Y. Generating functions for generalized Stirling type numbers, Array type polynomials, Eulerian type polynomials and their applications. Fixed Point Theory Appl. 2013, 87, 1-28. [CrossRef]

23. Simsek, Y. New families of special numbers for computing negative order Euler numbers and related numbers and polynomials. Appl. Anal. Discret. Math. 2018, 12,1-35. [CrossRef]

24. Simsek, Y. Explicit formulasfor $p$-adic integrals: Approach to $p$-adic distributions and some families of special numbers and polynomials. Montes Taurus J. Pure Appl. Math. 2019, 1, 1-76.

25. Simsek, Y. New classes of recurrence relations involving hyperbolic functions, special numbers and polynomials. Appl. Anal. Discrete Math. AADM 3138.

26. Simsek, Y.; Srivastava, H.M. A family of $p$-adic twisted interpolation functions associated with the modified Bernoulli numbers. Appl. Math. Comput. 2010, 216, 2976-2987. [CrossRef]

27. Simsek, Y.; Cangul, I.N.; Kurt, V.; Kim, D. $q$-Genocchi numbers and polynomials associated with $q$-Genocchi-type $l$-functions Adv. Differ. Equ. 2008, 815750. [CrossRef]

28. Simsek, Y.; Kim, D.; Rim, S.H. On the two-variable Dirichlet $q$-L-series. Adv. Stud. Contemp. Math. 2005, 10, 131-142.

29. Srivastava, H.M. Some generalizations and basic (or $q$-) extensions of the Bernoulli, Euler and Genocchi polynomials. Appl. Math. Inf. Sci. 2011, 5, 390-444.

30. Srivastava, H.M.; Choi, J. Series Associated with the Zeta and Related Functions; Kluwer Academic Publishers: Dordrecht, The Netherlands; Boston, MA, USA; London, UK, 2001.

31. Srivastava, H.M.; Choi, J. Zeta and q-Zeta Functions and Associated Series and Integrals; Elsevier Science Publishers: Amsterdam, The Netherlands; London, UK; New York, NY, USA, 2012.

32. Srivastava, H.M.; Kim, T.; Simsek, Y. $q$-Bernoulli numbers and polynomials associated with multiple $q$-zeta functions and basic L-series. Russ. J. Math. Phys. 2005, 12, 241-268.

33. Srivastava, H.M.; Saxena, R.K.; Pogany, T.K.; Saxena, R. Integral and computational representations of the extended HurwitzLerch zeta function. Integral Transform. Spec. Funct. 2011, 22, 487-506. [CrossRef]

34. Srivastava, H.M.; Ozden, H.; Cangul, I.N.; Simsek, Y. A unified presentation of certain meromorphic functions related to the families of the partial zeta type functions and the L-functions. Appl. Math. Comput. 2012, 219, 3903-3913. [CrossRef] 